\title{
A polarizable ion model for the structure of molten Cul
}

\author{
Vicente Bitrián, Olga Alcaraz, and Joaquim Trullàsa) \\ Departament de Física i Enginyeria Nuclear, Universitat Politècnica de Catalunya, \\ Campus Nord UPC B4-B5, 08034 Barcelona, Spain
}

(Received 8 October 2010; accepted 17 November 2010; published online 24 January 2011)

\begin{abstract}
The results are reported of the molecular dynamics simulations of the coherent static structure factor of molten $\mathrm{CuI}$ at $938 \mathrm{~K}$ using a polarizable ion model. This model is based on a rigid ion potential to which the many body interactions due to the anions induced polarization are added. The calculated structure factor reproduces the clear sharp prepeak observed in neutron diffraction data. The corresponding partial structure factors and the related radial distribution functions calculated by molecular dynamics are compared with those found in the literature derived from a combination of neutron and x-ray diffraction data with the aid of the reverse Monte Carlo simulation technique, as well as those calculated by ab initio MD simulations. () 2011 American Institute of Physics. [doi:10.1063/1.3525461]
\end{abstract}

\section{INTRODUCTION}

Copper iodide exhibits exceptionally high ionic (or superionic) conductivity in its solid phase, namely its ionic conductivity at high temperatures is of the same order as in the liquid phase $\left(\sim 1 \Omega^{-1} \mathrm{~cm}^{-1}\right){ }^{1,2}$ At ambient temperature and pressure $\mathrm{CuI}$ forms the $\gamma$ phase with zincblende structure where the $\mathrm{I}^{-}$ions occupy a face centered cubic (fcc) sublattice with the $\mathrm{Cu}^{+}$ions on another fcc sublattice shifted by $(1 / 4,1 / 4,1 / 4)$. At $642 \mathrm{~K}$ it undergoes a first order transformation to the $\beta$ phase with a structure similar to wurzite where the anions form a slightly distorted hexagonal close packed (hcp) sublattice and the cations are distributed over tetrahedral sites different from those of the standard wurzite structure. ${ }^{2}$ And at $680 \mathrm{~K}$ it experiences another first order transition to the superionic $\alpha$ phase, where the anions back to an fcc lattice as in the $\gamma$ phase, but the $\mathrm{Cu}^{+}$now occupy randomly four of the eight tetrahedral sites in the unit cell. Both $\beta$ and $\alpha$ phases have a high ionic conductivity due to the mobile $\mathrm{Cu}^{+},{ }_{3}^{3}$ which can jump between different sites. From the $\alpha$ phase $\mathrm{CuI}$ melts at $878 \mathrm{~K}$. The first order structural transitions $\gamma \rightarrow \beta$ and $\beta \rightarrow \alpha$ prevent a continuous change from the ambient to the superionic phase. ${ }^{1}$ This is the reason why $\mathrm{CuI}$ is included by Boyce and Huberman ${ }^{1}$ in the type $\mathrm{I}$ of superionic conductors whose paradigmatic example is $\alpha$-AgI since it experiences a sudden increase in its ionic conductivity of around 3 orders of magnitude at $420 \mathrm{~K}$ known as the $\beta \rightarrow \alpha$ transition.

The superionic behavior of $\alpha$-AgI and $\alpha$-CuI was reproduced by Vashishta and Rahman ${ }^{4,5}$ by molecular dynamics (MD) simulations using simple effective pair potentials. The two main characteristics of these rigid ion potentials, which we will denote as VR, are: (i) the absolute value of effective charges is lower than $e$ (the fundamental charge) to mimic the effect of covalent interactions, and (ii) the anionic radius is

\footnotetext{
a) Author to whom correspondence should be addressed. Electronic mail: quim.trullas@upc.edu.
}

about the double than the cationic one. By using a refined parameterization of the original VR potential, Parrinello et al. ${ }^{6}$ (PRV) carried out MD simulations of the $\beta \rightarrow \alpha$ transition in AgI. This particular VR potential, with the refined parameter values, has been widely used to study solid AgI. ${ }^{7-9}$ Furthermore, Stafford and Silbert ${ }^{10}$ carried out theoretical calculations of the radial distribution functions and structure factors of molten $\mathrm{AgI}$ within the hypernetted chain approximation by using the PRV potential. Their results turned out to qualitatively agree with the experimental coherent static structure factor $S(k)$ available shortly after ${ }^{11}$ despite some differences in the quantitative sense. Moreover, MD simulations of the ionic transport properties using the PRV potential suggested that molten AgI, near melting, retains the superionic character of the $\alpha$ phase. ${ }^{12,13}$ However, the calculated $S(k)$ failed to reproduce the almost featureless broad main peak between the shoulder at $1.8 \AA^{-1}$ and the maximum at around $2.8 \AA^{-1}$, as well as the prepeak that appears at around $1 \AA^{-1},{ }^{14}$ exhibited by the neutron diffraction (ND) data obtained by Inui et al., ${ }^{15}$ Shirakawa et al., ${ }^{16}$ and Kawakita et al. ${ }^{17,18}$ In fact, at first glance the prepeak appears as a shoulder, but there is a small maximum at around $1 \AA^{-1}$. More recently, Bitrian et al. ${ }^{19-22}$ showed that the inclusion of the anions polarizability in the VR potentials accounts for the experimental prepeak of the $S(k)$ for molten AgI, as well as improves the experimental almost featureless broad main peak. Actually, Bitrian et al. use the potential parameter values proposed by Shimojo and Kobayashi, ${ }^{23}$ who slightly modified the PRV parameterization in order to reproduce the $\alpha$-phase features at the appropriate experimental density.

Concerning molten CuI, the VR rigid ion pair potentials also fail to reproduce the clear sharp prepeak at around $0.9 \AA^{-1}$ in the experimental $S(k) .{ }^{24}$ Nevertheless, they are able to predict the main peak position at around $3 \AA^{-1}$ and that of the intermediate peak at around $1.75 \AA^{-1}$ observed in ND data. ${ }^{24-26}$ In the present paper we will show that the inclusion of the anions polarizability in the VR potentials accounts for the main features of the experimental $S(k)$ for molten CuI, 
$\mathrm{S}(\mathrm{k})$

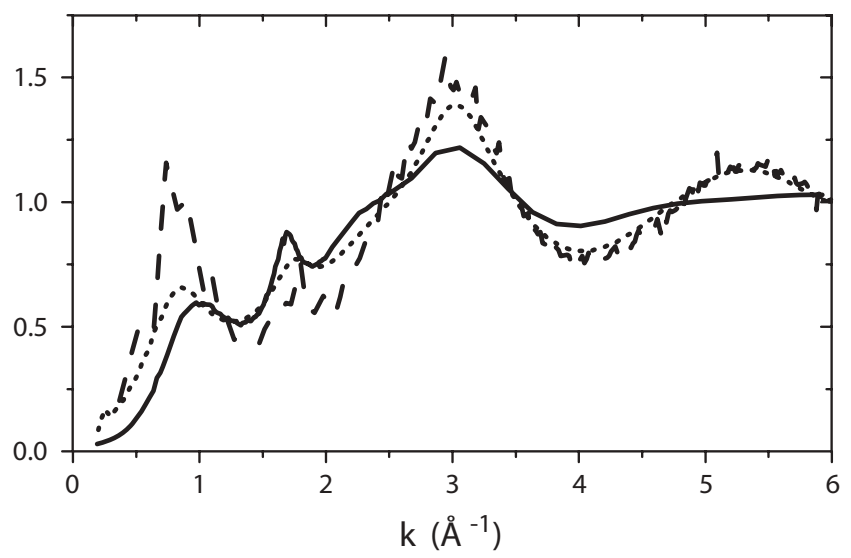

FIG. 1. Coherent static structure factors, $S(k)$, for molten CuI from ND experimental data by Drewitt et al. (Ref. 24) at $933 \mathrm{~K}$ (dotted line), MD results of this work at $938 \mathrm{~K}$ for the PIM (solid line) and $a b$ initio MD results by Shimojo et al. (Ref. 38) at $900 \mathrm{~K}$ (dashed line).

including the first sharp diffraction peak (see Fig. 1). The layout of this paper is as follows. We describe the polarizable ion model (PIM) and give some details of MD simulations in Sec. II. We present and discuss the results in Sec. III, and summarize them in the concluding remarks of Sec. IV.

\section{POLARIZABLE ION MODEL AND MD SIMULATIONS DETAILS}

The VR rigid ion pair potentials proposed originally in Ref. 5 can be written as

$$
\phi_{a b}(r)=\phi_{a b}^{0}(r)-\frac{P_{a b}}{r^{4}}
$$

with

$$
\phi_{a b}^{0}(r)=\frac{z_{a} z_{b} e^{2}}{r}+\frac{H_{a b}}{r^{7}}-\frac{C_{a b}}{r^{6}} .
$$

The first term on the rhs of Eq. (2) is the Coulomb interaction between the charges, with $\left|z_{a}\right|=0.6 e$ the effective charge for cations and anions; the second models the repulsion between the ions, with $H_{a b}=A\left(\sigma_{a}+\sigma_{b}\right)^{7}$, where $\sigma_{\mathrm{Cu}}$ $=0.482 \AA$ and $\sigma_{\mathrm{I}}=2.135 \AA$ are related to the ionic radii, and $A=0.222 \mathrm{eV}$ defines the strength of the repulsive interactions. The third term is the van der Waals contribution, with $C_{a b}=(3 / 2) \alpha_{a} \alpha_{b}\left(E_{a}^{-1}+E_{b}^{-1}\right)^{-1}$, where $\alpha_{a}$ are the polarizabilities and $E_{a}$ are related to the ionization potentials of the cations and electron affinities of the anions. The second term on the rhs of Eq. (1) denotes the pairwise effective monopoleinduced dipole interaction with $P_{a b}=(1 / 2)\left(\alpha_{a} z_{b}^{2}+\alpha_{b} z_{a}^{2}\right) e^{2}$. Then, assuming $\alpha_{\mathrm{Cu}}=0$, it leads to $C_{\mathrm{CuCu}}=C_{\mathrm{CuI}}=P_{\mathrm{CuCu}}$ $=0$. The value given in Refs. 4 and 5 for $C_{\mathrm{II}}$ is $99.8 \mathrm{eV} \AA^{6}$. Hereafter we will refer to this model as the rigid ion model (RIM).

The polarizable ion model used in this work is constructed by adding the induced polarization contributions to the pair potential $\phi_{a b}^{0}(r)$ as in Ref. 27 . We assume that, on an ion placed at position $\mathbf{r}_{i}$, the local electric field $\mathbf{E}_{i}$ due to all the other ions induces a point dipole whose moment is

$$
\boldsymbol{\mu}_{i}=\alpha_{i} \mathbf{E}_{i}=\alpha_{i}\left(\mathbf{E}_{i}^{\mathrm{q}}+\mathbf{E}_{i}^{\mu}\right),
$$

where $\mathbf{E}_{i}^{\mathrm{q}}$ is the field at $\mathbf{r}_{i}$ due to all the point charges except $q_{i}=z_{i} e$, and $\mathbf{E}_{i}^{\mu}$ the field at $\mathbf{r}_{i}$ due to all the dipole moments except $\boldsymbol{\mu}_{i}$. The potential energy of this PIM may be written as

$$
\begin{aligned}
U= & \frac{1}{2} \sum_{i=1}^{N} \sum_{j \neq i}^{N} \phi_{i j}^{0}\left(r_{i j}\right)-\sum_{i=1}^{N} \boldsymbol{\mu}_{i} \cdot \mathbf{E}_{i}^{q} \\
& -\frac{1}{2} \sum_{i=1}^{N} \boldsymbol{\mu}_{i} \cdot \mathbf{E}_{i}^{\mu}+\sum_{i=1}^{N} \frac{\mu_{i}^{2}}{2 \alpha_{i}} .
\end{aligned}
$$

The original value for $\alpha_{\text {I }}$ used in Refs. 4-6 was $6.52 \AA^{3}$. This value is too large since it leads to the polarization catastrophe in the MD simulations of the corresponding PIM for $\mathrm{CuI}$ (but not AgI), i.e., two iodides can approach at unphysical distances and they become overpolarized. ${ }^{28,29}$ However, with $\alpha_{\mathrm{I}}=6.12 \AA^{3}$, as it was proposed by Shimojo and Kobayashi for molten $\mathrm{AgI}$, the polarization catastrophe in molten $\mathrm{CuI}$ is avoided. The MD results presented in this work have been obtained with this lower value. It is possible to construct other PIM with a short-range damping polarizability that opposes the electrically induced dipole moments; these are discussed in Refs. 20 and 30. However, when the short damping polarizability is used to avoid the polarization catastrophe with $\alpha_{\mathrm{I}}$ $=6.52 \AA^{3}$, the range of the damping must be too long and the polarization effects are so damped that the results become close to those for the RIM.

In the present work we have carried out MD simulations of the RIM and PIM over $3 \times 10^{5}$ time steps, with a time step $\Delta t=5 \times 10^{-15} \mathrm{~s}$, using $N=1000$ ions placed in a cubic box of side $L$ with an ionic density $\rho=N / L^{3}=0.02813 \AA^{-3}$. The temperature is $938 \mathrm{~K}$, very close to the $933 \mathrm{~K}$ at which ND experimental data of Drewitt et al. ${ }^{24}$ were measured. According to Janz et $a l .{ }^{31}$ the ionic density at $938 \mathrm{~K}$ is that used in this work. Computational details, as well as the prescription to calculate the structure factors, are described in Refs. 20, 27, and 30. From the Ashcroft-Langreth partial structure factors, $S_{a b}(k)$, we have calculated the coherent static structure factor, $S(k)=(1 / 2)\left[b_{\mathrm{Cu}}^{2} S_{\mathrm{CuCu}}(k)+2 b_{\mathrm{Cu}} b_{\mathrm{I}} S_{\mathrm{CuI}}(k)+b_{\mathrm{I}}^{2} S_{\mathrm{II}}(k)\right] / b^{2}$ with $b^{2}=\left(b_{\mathrm{Cu}}^{2}+b_{\mathrm{I}}^{2}\right) / 2$ (Ref. 32), using the neutron scattering lengths values $b_{\mathrm{Cu}}=7.718 \mathrm{fm}$ and $b_{\mathrm{I}}=5.280 \mathrm{fm}^{33}$

\section{RESULTS AND DISCUSSION}

The experimental ND data of $S(k)$ for molten CuI, with which we compare our simulation results, are the results of Drewitt et $a l .^{24}$ In fact they reported $F(k)=b^{2}[S(k)-1]$ in terms of the Faber-Zimman partial structure factors $A_{a b}(k)=\left(c_{a} c_{b}\right)^{-1 / 2}\left[S_{a b}(k)-\delta_{a b}\right]+1 . .^{34,35}$ Their ND data exhibit the same trends as those published much earlier by Shirakawa et al. ${ }^{25}$ at 938 and $1033 \mathrm{~K}$, and those more recent by Takeda et al. ${ }^{26}$ at $923 \mathrm{~K}$. The values of the $S(k)$ by Shirakawa et al. at $938 \mathrm{~K}$ are slightly lower than those of Drewitt et al. and Takeda et al., which are very similar although the peak positions of the latter are slightly shifted at higher wave 
numbers. Takeda et al., with the aid of the reverse Monte Carlo (RMC) simulation technique, derived the corresponding partial structure factors $S_{a b}(k)$ and the related radial distribution functions $g_{a b}(r)$, from a combination of neutron and $\mathrm{x}$-ray diffraction data. These functions were also published by Kawakita et al. ${ }^{18}$ The functions $S_{a b}(k)$ and $g_{a b}(r)$ were also estimated with the RMC from anomalous X-ray scattering by Waseda et al. ${ }^{36}$ at $940 \mathrm{~K}$, and the short-range part of $g_{a b}(r)$ was also measured using X-ray absorption spectroscopy by Trapananti et al. ${ }^{37}$ at $903 \mathrm{~K}$. Furthermore, Shimojo et al. ${ }^{38}$ carried out $a b$ initio MD simulations of molten CuI at $900 \mathrm{~K}$ and calculated $S(k), S_{a b}(k)$, and $g_{a b}(r)$. Comparison between all these results is made in the paper of Drewitt et al. ${ }^{24}$ [with $F(k)$ and $A_{a b}(k)$ in place of $S(k)$ and $S_{a b}(k)$, respectively].

In Fig. 1 the $S(k)$ results of our PIM simulations are compared with the ND experimental data of Drewitt et al., together with the $a b$ initio MD results of Shimojo et al. The PIM $S(k)$ reproduces the sharp prepeak at around $0.9 \AA^{-1}$ in the ND $S(k)$, which is also found much higher and at a slightly lower wave number in the $a b$ initio $S(k)$. The main peak at around $3 \AA^{-1}$ is lower for PIM than that for ND and ab initio, while the intermediate small peak at around $1.75 \AA^{-1}$ for PIM is slightly higher than the other two, but much lower than that

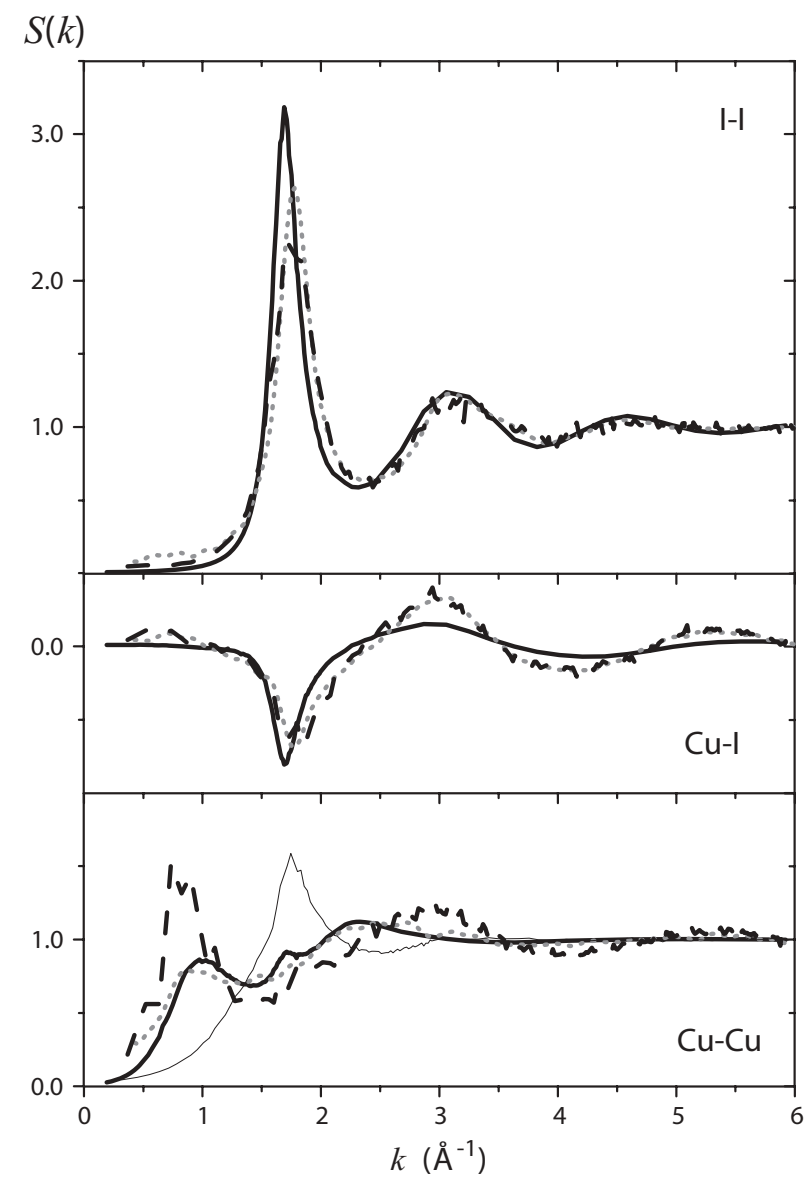

FIG. 2. Ashcroft-Langreth partial structure factors for molten $\mathrm{CuI}, S_{\mathrm{II}}(k)$ (top), $S_{\mathrm{CuI}}(k)$ (middle), and $S_{\mathrm{CuCu}}(k)$ (bottom), from RMC of a combination of neutron and X-ray diffraction data by Kawakita et al. (Ref. 18) at $923 \mathrm{~K}$ (dotted line), MD results of this work at $938 \mathrm{~K}$ for the PIM (solid line) and ab initio MD results by Shimojo et al. (Ref. 38) at $900 \mathrm{~K}$ (dashed line). The thin solid line in the $S_{\mathrm{CuCu}}(k)$ is that obtained for the RIM. obtained using the RIM (see Ref. 24). Moreover, the PIM $S(k)$ oscillations beyond the main peak damp faster.

The $S_{a b}(k)$ and $g_{a b}(r)$ calculated from PIM simulations are shown in Figs. 2 and 3, respectively, where they are compared with those given by Kawakita et al. ${ }^{18}$ from a combination of neutron and $\mathrm{x}$-ray diffraction data with the aid of RMC, as well as those obtained from $a b$ initio MD simulations by Shimojo et al. ${ }^{38}$ For the sake of clarity, in these figures we have not plotted, but commented, the corresponding

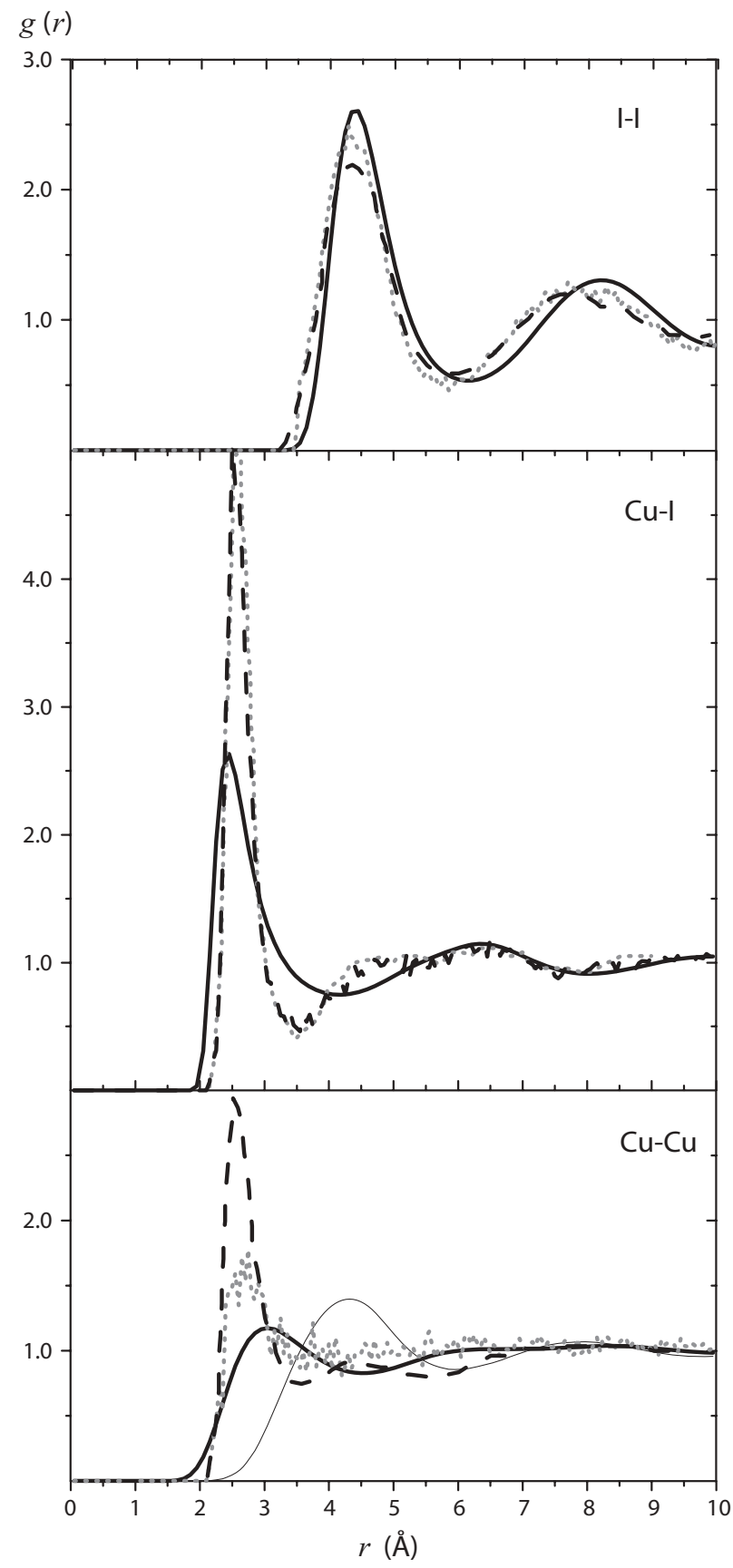

FIG. 3. Radial distribution functions for molten CuI, $g_{\mathrm{II}}(r)$ (top), $g_{\mathrm{CuI}}(r)$ (middle), and $g_{\mathrm{CuCu}}(r)$ (bottom), from RMC of a combination of neutron and X-ray diffraction data by Kawakita et al. (Ref. 18) at $923 \mathrm{~K}$ (dotted line), MD results of this work at $938 \mathrm{~K}$ for the PIM (solid line) and ab initio MD results by Shimojo et al. (Ref. 38) at $900 \mathrm{~K}$ (dashed line). The thin solid line in the $g_{\mathrm{CuCu}}(r)$ is that obtained for the RIM. 
RIM results, except in the $\mathrm{Cu}-\mathrm{Cu}$ correlations case where the differences are more significant.

The PIM, RMC, and ab initio $S_{\mathrm{II}}(k)$ differ only in the height of the main peak at around $k_{\mathrm{M}} \approx 1.75 \AA^{-1}$, the same position of the intermediate small peak in $S(k)$, with that for PIM higher than that from $a b$ initio MD and that from RMC in between. In the real space, the corresponding $g_{\mathrm{II}}(r)$ are almost identical with the main peak at around $4.4 \AA$, although the first peak in the PIM $g_{\mathrm{II}}(r)$ is slightly higher than that from $a b$ initio, with the height from RMC in between. Moreover, in the PIM case, the wavelength of the $g_{\text {II }}(r)$ oscillations beyond the first peak is slightly longer, and the values before the first peak are slightly lower. Nevertheless, the wavelength of the oscillations, which can be estimated from the distance between the first and second peaks of $g_{\mathrm{II}}(r)$, is in the three cases close to $\lambda \approx 3.6 \AA$, whose wave number is $k_{\mathrm{M}} \approx 2 \pi / \lambda$ $\approx 1.75 \AA^{-1}$. The $S_{\mathrm{II}}(k)$ and $g_{\mathrm{II}}(r)$ from RIM are very close to that from PIM, this is the reason why we do not plotted these results for the RIM case. It is interesting to note that, although we only take into account the anion polarizability, it is the PIM cation-cation structure that is mainly affected by the induced polarization interactions, as we will see below, while the anion-anion structure is practically unaffected. Since the iodides are much bigger than copper cations and, thus, they are much closely packed, it appears that their structure is mainly determined by the way how they can be placed in the available space.

In spite of small differences, the PIM, RMC, and ab initio $S_{\text {CuI }}(k)$ exhibit the same trends, with a first valley at around $k_{\mathrm{M}}$ $\approx 1.75 \AA^{-1}$ slightly deeper and shifted at lower values for the PIM case. The RMC and $a b$ initio $g_{\mathrm{CuI}}(r)$ are almost identical with the first peak at around $2.6 \AA$ much higher than that for the PIM $g_{\mathrm{CuI}}(r)$, which is slightly shifted at a lower position. Moreover, the wavelength of the PIM $g_{\mathrm{CuI}}(r)$ oscillations beyond the first peak is longer. If comparison is made between the PIM and RIM results, they are similar but with the first peak of the latter higher, and their oscillations slightly more pronounced, than those for the former.

The main differences between the RIM, PIM, RMC, and $a b$ initio results are found in the $\mathrm{Cu}-\mathrm{Cu}$ correlations. At a first sight, the qualitative behavior of each $S_{\mathrm{CuCu}}(k)$ reminds that of the corresponding $S(k)$. It is because the neutron scattering length for $\mathrm{Cu}^{+}(7.718 \mathrm{fm})$ is larger than for $\mathrm{I}^{-}(5.280 \mathrm{fm})$, and the contribution $b_{\mathrm{Cu}}^{2} S_{\mathrm{CuCu}}(k)$ in $S(k)$ prevails over those of $2 b_{\mathrm{Cu}} b_{\mathrm{I}} S_{\mathrm{CuI}}(k)$ and $b_{\mathrm{I}}^{2} S_{\mathrm{II}}(k)$ which almost cancel each other. The most salient feature in the PIM, RMC, and $a b$ initio $S_{\mathrm{CuCu}}(k)$ is the clear prepeak approximately at about $0.9 \AA^{-1}$ not present in the RIM, with that from ab initio at around $0.7 \AA^{-1}$ and that for the PIM at $1 \AA^{-1}$. The PIM, RMC, and $a b$ initio $S_{\mathrm{CuCu}}(k)$ also exhibit, as the three $S(k)$, the intermediate small peak at around $k_{\mathrm{M}} \approx 1.75 \AA^{-1}$ before the higher peak, while the RIM $S_{\mathrm{CuCu}}(k)$ only presents a first peak at around $k_{\mathrm{M}} \approx 1.75 \AA^{-1}$. It is because the RIM $g_{\mathrm{CuCu}}(r)$ oscillates with the same wavelength as $g_{\mathrm{II}}(r)$, while this periodicity is in some sense lost in the $g_{\mathrm{CuCu}}(r)$ obtained from PIM, RMC, and ab initio simulations.

Regarding the PIM, RMC, and $a b$ initio $g_{\mathrm{CuCu}}(r)$, the first peak in the three functions is about the same position of that in $g_{\mathrm{CuI}}(r)$, with that from $a b$ initio higher and at a lower

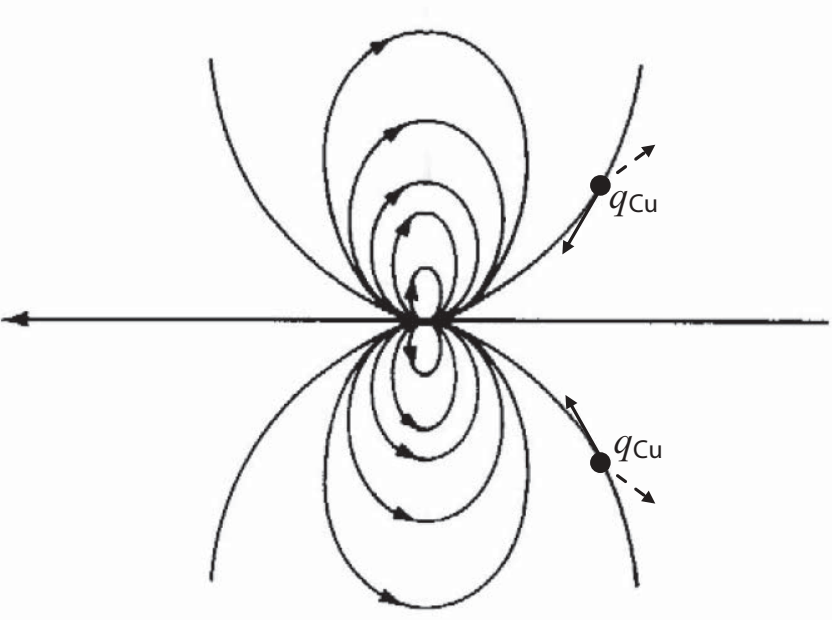

FIG. 4. Two copper cations under the electric field action of an induced iodide point dipole. The solid arrows are the force of this field over each cation. The dashed arrows are the strong short-range radial repulsion force of the iodide over each copper ion.

position (at around $2.5 \AA$ ) than that from RMC (at around $2.6 \AA$ ), and that for PIM the lowest, less sharp and at a larger position (at around $3 \AA$ ), in such a way that the three $g_{\mathrm{CuCu}}(r)$ are not in phase with $g_{\mathrm{II}}(r)$, neither in opposite phase with $g_{\mathrm{CuI}}(r)$, as in the RIM case and the typical structure of molten alkali halides. Moreover, the PIM $g_{\mathrm{CuCu}}(r)$ presents a second small maximum at around $6.3 \AA$, while that from $a b$ initio is at around $4.4 \AA$. Since these positions are lower than (or equal to) the position of the second peak in $g_{\mathrm{CuI}}(r)$, it means that around each copper cation there is a double shell of cations between the first and second shells of iodides. Then, the weak oscillations in $g_{\mathrm{CuCu}}(r)$ do not repeat themselves with a clear wavelength. This is the reason why $S_{\mathrm{CuCu}}(k)$ exhibits the higher peak beyond the intermediate small peak at $k_{\mathrm{M}} \approx 1.75 \AA^{-1}$.

Comparison between the PIM and RIM $g_{\mathrm{CuCu}}(r)$ shows that the first peak of the PIM $g_{\mathrm{CuCu}}(r)$ is shifted inwards with a deeper cations penetration, i.e., the separation between neighboring cations can be smaller than it would be the case if the anions were not polarized. This feature may be attributed to the screening of the cations repulsion due to the anion induced dipoles. This effect may be illustrated in Fig. 4 where can be seen the electric field lines of an iodide point dipole induced by two equidistant copper cations and the force of this field over each cation (solid arrows). However, the strong shortrange radial repulsion force of the iodide over the copper ions (dashed arrows) due to the term $H_{+} / r^{7}$ in Eq. (2) avoids that the latter approach to the former, and the overall effect is that the two copper cations approach to each other, in such a way that low cation density regions, also called cation voids, are opened. The length scale which characterizes the ordering of the voids between cations is related to the prepeak at about $0.9 \AA^{-1}$ in the corresponding $S_{\mathrm{CuCu}}(k)$, or $S(k)$.

The prepeak is a signature of intermediate range order. Since it is the result of subtle correlations with wavelength about $2 \pi /\left(0.9 \AA^{-1}\right) \approx 7 \AA$, its structural origin is difficult to distinguish in $g_{\mathrm{CuCu}}(r)$, namely the differences between the inverse Fourier transform of $\mathrm{S}_{\mathrm{CuCu}}(k)$ with and without 
the prepeak are barely perceptible. For instance, the behavior of the $g_{\mathrm{AgAg}}(r)$ calculated from MD of a PIM based on VR potentials for molten $\mathrm{AgCl}$ (see Ref. 27) is very similar to that of the PIM $g_{\mathrm{CuCu}}(r)$, but the corresponding $S_{\mathrm{AgAg}}(k)$ does not present any prepeak. Then, as in our previous paper on molten AgI, ${ }^{19}$ to gain more insight into the intermediaterange order related to the prepeak, and following the idea suggested by Madden and Wilson, ${ }^{39,40}$ we have applied the Voronoi-Delaunay method. ${ }^{41,42}$ This method measures the empty space between tetrahedras of four cations at the vertices, with no other cation centre lying within its circumsphere, in such a way that the tetrahedras fills the space without any gap. With this method we have studied the cation-void configurations and we have compared the void radii distribution, $D\left(R_{\mathrm{V}}\right)$, and the void-void structure factor $S_{V V}(k)$ for the PIM and RIM. The results are very similar to those found for molten AgI. $D\left(R_{\mathrm{V}}\right)$ shows that the averaged cation configuration in the PIM has bigger empty spaces (voids), and comprises a wider variety of small and big voids, than in RIM. In addition, the PIM $S_{\mathrm{VV}}(k)$ has a very intense peak at around $1.1 \AA^{-1}$, very close to the $S_{\mathrm{CuCu}}(k)$ prepeak (around $1 \AA^{-1}$ ), while that for the RIM presents the peak at a wave number close to the peak of the RIM $S_{\mathrm{CuCu}}(k)$ at around $1.75 \AA^{-1}$. Hence we conclude that the prepeaks in $S(k)$ and $S_{\mathrm{CuCu}}(k)$ for the PIM appears to be the signature of a periodicity of low cation density zones (or voids), due to anion polarization effects.

In the light of the above results, as well as those from MD simulations of molten AgI using PIM, ${ }^{19,20}$ it seems clear that anion polarizability is in the origin of the $S(k)$ prepeak. This agrees with the fact that the prepeak in the ND $S(k)$ for molten $\mathrm{CuBr}$ is lower than that for molten $\mathrm{CuI}$ (see Ref. 25) because $\alpha_{\mathrm{Br}}<\alpha_{\mathrm{I}}$.

\section{CONCLUDING REMARKS}

Summing up, we have shown that the main trends of the experimental coherent static structure factor of molten CuI, especially the prepeak at around $0.9 \AA^{-1}$, can be reproduced by adding the anion polarizability to simple rigid ion pair potentials of the Vashishta and Rahman form, ${ }^{4,5}$ by using an anion polarizability $\alpha_{\mathrm{I}}=6.12 \AA^{3}$, as it was proposed by Shimojo and Kobayashi for AgI, ${ }^{23}$ lower than the original value $\alpha_{\mathrm{I}}=6.52 \AA^{3}$ used in Ref. 5, which leads to the polarization catastrophe in MD simulations of molten CuI. Furthermore, we have also shown that the main trends of the corresponding partial structure factors and the related radial distribution functions agree with those derived from a combination of neutron and $\mathrm{x}$-ray diffraction data with the aid of the reverse Monte Carlo simulation technique by Takeda et al. ${ }^{26}$ and Kawakita et al. ${ }^{18}$ as well as those calculated from ab initio MD simulations by Shimojo et al. ${ }^{38}$ We are now working on MD simulations of molten $\mathrm{CuBr}$ and $\mathrm{CuCl}$ using polarizable ion models. We expect to publish a larger paper than the present one to report the results for the structure and ionic transport properties of molten copper halides.

\section{ACKNOWLEDGMENTS}

We thank Phil Salmon because he made available the tabulated data of their experimental ND data for molten CuI in Ref. 24, which also included the $a b$ initio MD results of Shimojo et al. in Ref. 38. We also thank Yukinobu Kawakita for the tabulated data of their RMC results in Ref 18 . This work was supported by DGI of Spain (Grant No. FIS2009-13641-C02-01) and the DURSI of the Generalitat of Catalonia (Grant No. 2009SGR-1003).

${ }^{1}$ J. B. Boyce and B. A. Huberman, Phys. Rep. 51, 189 (1979).

${ }^{2}$ S. Hull, Rep. Prog. Phys. 67, 1233 (2004).

${ }^{3}$ A. Chaid and R. L. McGreevy, J. Phys.: Condens. Matter 10, 2597 (1998).

${ }^{4}$ P. Vashishta and A. Rahman, Phys. Rev. Lett. 40, 1337 (1978).

${ }^{5}$ P. Vashishta and A. Rahman, Fast Ion Transport in Solids, edited by P. Vashishta, J. N. Mundy, and G. K. Shenoy (North-Holland, Amsterdam, 1979).

${ }^{6}$ M. Parrinello, A. Rahman, and P. Vashishta, Phys. Rev. Lett. 50, 1073 (1983).

${ }^{7}$ J. L. Tallon, Phys. Rev. B 38, 9069 (1988).

${ }^{8}$ C. Seok and D. W. Oxtoby, Phys. Rev. B 56, 11485 (1997).

${ }^{9}$ D. A. Keen, S. Hull, A. C. Barnes, P. Berastegui, W. A. Crichton, P. A. Madden, M. G. Tucker, and M. Wilson, Phys. Rev. B 68, 014117 (2003).

${ }^{10}$ A. J. Stafford and M. Silbert, Z. Phys. B 67, 31 (1987).

${ }^{11}$ H. Takahashi, S. Takeda, S. Harada, and S. Tamaki, J. Phys. Soc. Jpn. 57, 562 (1988).

${ }^{12}$ A. J. Stafford, M. Silbert, J. Trullàs, and A. Giró, J. Phys.: Condens. Matter 2, 6631 (1990). (In this article the curves should be interchanged between Figs. 2 and 3 in order to match the figure captions. Moreover, in Table I the value of $\alpha_{\chi}$ for $\mathrm{AgI}$ should be $6.52 \AA^{3}$ and that of $H_{\mathrm{XX}}$ for $\mathrm{CuI}$ should be 399 in energy units of $e^{2} / \AA=14.4 \mathrm{eV}$ as in Ref. 5.)

${ }^{13}$ J. Trullàs, A. Giró, and M. Silbert, J. Phys.: Condens. Matter 2, 6643 (1990).

${ }^{14}$ V. Bitrián, J. Trullàs, M. Silbert, T. Enosaki, Y. Kawakita, and S. Takeda, J. Chem. Phys. 125, 184510 (2006).

${ }^{15}$ M. Inui, S. Takeda, Y. Shirakawa, S. Tamaki, Y. Waseda, and Y. Yamaguchi, J. Phys. Soc. Jpn. 60, 3025 (1991).

${ }^{16}$ Y. Shirakawa, S. Tamaki, T. Usuki, K. Sugiyama, and Y. Waseda, J. Phys. Soc. Jpn. 63, 1814 (1994).

${ }^{17}$ Y. Kawakita, T. Enosaki, S. Takeda, and K. Maruyama, J. Non-Cryst. Solids 353, 3035 (2007).

${ }^{18}$ Y. Kawakita, S. Tahara, H. Fujii, S. Kohara, and S. Takeda, J. Phys.: Condens. Matter 19, 335201 (2007).

${ }^{19}$ V. Bitrián, J. Trullàs, and M. Silbert, J. Chem. Phys. 126, 021105 (2007).

${ }^{20}$ V. Bitrián and J. Trullàs, J. Phys. Chem. B 112, 1718 (2008).

${ }^{21}$ V. Bitrián and J. Trullàs, J. Phys.: Conf. Ser. 98, 042006 (2008).

${ }^{22}$ V. Bitrián, O. Alcaraz, and J. Trullàs, J. Chem. Phys. 130, 234504 (2009).

${ }^{23}$ F. Shimojo and M. Kobayashi, J. Phys. Soc. Jpn. 60, 3725 (1991).

${ }^{24}$ J. E. Drewitt, P. S. Salmon, S. Takeda, and Y. Kawakita, J. Phys.: Condens. Matter 21, 075104 (2009).

${ }^{25}$ Y. Shirakawa, M. Saito, S. Tamaki, M. Inui, and S. Takeda, J. Phys. Soc. Jpn. 60, 2678 (1991)

${ }^{26}$ S. Takeda, H. Fujii, Y. Kawakita, Y. Kato, S. Kohara, and K. Maruyama, Physica B 385-386, 249 (2006).

${ }^{27}$ J. Trullàs, O. Alcaraz, L. E. González, and M. Silbert, J. Phys. Chem. B 107, 282 (2003).

${ }^{28}$ M. Wilson and P. A. Madden, J. Phys.: Condens. Matter 5, 2687 (1993).

${ }^{29}$ O. Alcaraz, V. Bitrián, and J. Trullàs, J. Chem. Phys. 127, 154508 (2007).

${ }^{30}$ V. Bitrián and J. Trullàs, J. Phys. Chem. B 110, 7490 (2006).

${ }^{31}$ G. J. Janz, F. W. Dampier, G. R. Lakshminarayanan, P. K. Lorenz, and R. P. T. Tomkins, Molten Salts: Volume 1. Electrical Conductance, Density, and Viscosity Data, National Standard Reference Data Series-National Bureau of Standards 15 (U.S. Government Printing Office, Washington, DC, 1968)

${ }^{32}$ M. P. Tosi, D. L. Price, and M.-L. Saboungi. Annu. Rev. Phys. Chem. 44, 173 (1993). 
${ }^{33}$ V. F. Sears, Neutron News 3, 26 (1992).

${ }^{34}$ H. E. Fisher, A. C. Barnes, and P. S. Salmon, Rep. Prog. Phys. 69, 233 (2006).

${ }^{35}$ M. Rovere and M. P. Tosi, Rep. Prog. Phys. 49, 1001 (1986).

${ }^{36}$ Y. Waseda, S. Kang, K. Sugiyama, M. Kimura, and M. Saito, J. Phys.: Condens. Matter 12, A195 (2000).

${ }^{37}$ A. Trapananti, A. Di Cicco, and M. Minicucci, Phys. Rev. B 66, 014202 (2002).
${ }^{38}$ F. Shimojo, M. Aniya, and K. Hoshino, J. Phys. Soc. Jpn. 73, 2148 (2004).

${ }^{39}$ P. A. Madden and M. Wilson, J. Phys.: Condens. Matter 12, A95 (2000).

${ }^{40}$ M. Wilson and P. A. Madden, Phys. Rev. Lett. 80, 532 (1998).

${ }^{41}$ Y. I. Naberukhin, V. P. Voloshin, and N. N. Medvedev, Mol. Phys. 73, 917 (1991).

${ }^{42}$ M. Tanemura, T. Ogawa, and N. Ogita, J. Comput. Phys. 51, 191 (1983). 